\title{
Inactivating Anterior Insular Cortex Reduces Risk Taking
}

\author{
Hironori Ishii, ${ }^{1}$ Shinya Ohara, ${ }^{1}$ Philippe N. Tobler, ${ }^{2}$ Ken-Ichiro Tsutsui, ${ }^{1}$ and Toshio Iijima ${ }^{1}$ \\ ${ }^{1}$ Division of Systems Neuroscience, Tohoku University Graduate School of Life Sciences, Sendai 980-8577, Japan, and ${ }^{2}$ Laboratory for Social and Neural \\ Systems Research, Department of Economics, University of Zurich, 8006 Zürich, Switzerland
}

We often have to make risky decisions between alternatives with outcomes that can be better or worse than the outcomes of safer alternatives. Although previous studies have implicated various brain regions in risky decision making, it remains unknown which regions are crucial for balancing whether to take a risk or play it safe. Here, we focused on the anterior insular cortex (AIC), the causal involvement of which in risky decision making is still unclear, although human imaging studies have reported AIC activation in various gambling tasks. We investigated the effects of temporarily inactivating the AIC on rats' risk preference in two types of gambling tasks, one in which risk arose in reward amount and one in which it arose in reward delay. As a control within the same subjects, we inactivated the adjacent orbitofrontal cortex (OFC), which is well known to affect risk preference. In both gambling tasks, AIC inactivation decreased risk preference whereas OFC inactivation increased it. In risk-free control situations, AIC and OFC inactivations did not affect decision making. These results suggest that the AIC is causally involved in risky decision making and promotes risk taking. The AIC and OFC may be crucial for the opposing motives of whether to take a risk or avoid it.

\section{Introduction}

The choice of taking a risk for a higher gain or playing it safe and thereby avoiding a loss is an important one. Risk taking can lead to ruin but may also prove advantageous by facilitating exploration. The point is their balance. Many behavioral studies have investigated decision making under risk in humans (Tversky and Kahneman, 1981) and animals (for review, see Kacelnik and Bateson, 1996; Weber et al., 2004) and have shown that decisions made by a wide variety of species are sensitive to the balance of risky and sure outcomes.

Recent studies have elucidated the neural basis underlying decision making under risk (for review, see Doya, 2008; Rushworth et al., 2011; Schultz, 2011). A key region is the anterior insular cortex (AIC). In human neuroimaging studies, the AIC was activated during decision making under risk in various gambling tasks (Paulus et al., 2003; Kuhnen and Knutson, 2005; Preuschoff et al., 2008; Xue et al., 2010). For example, insular activation was stronger when participants chose risky alternatives versus sure alternatives (Paulus et al., 2003). AIC activity was correlated with risk prediction and risk prediction error (Preuschoff et al., 2008), and reflected higher forms of risk (Burke and Tobler, 2011). However, whether AIC causally contributes to decision making under risk is largely unknown.

\footnotetext{
Received May 11, 2012; revised Sept. 10, 2012; accepted Sept. 14, 2012.

Author contributions: H.I., S.O., P.N.T., K.-I.T., and T.I. designed research; H.I. performed research; H.I. analyzed data; H.I., S.O., P.N.T., K.-I.T., and T.I. wrote the paper.

This work was supported by a Global Common Operating Environment Program of the Japanese Ministry of Education, Culture, Sports, Science and Technology in the form of the "Basic and Translational Research Center for Global Brain Science," at Tohoku University.

The authors declare no financial conflicts of interest.

Correspondence should be addressed to Dr. Toshio lijima, Division of Systems Neuroscience, Tohoku University Graduate School of Life Sciences, 2-1-1 Katahira, Aoba-ku, Sendai 980-8577, Japan. E-mail: t-iijima@ m.tohoku.ac.jp.

DOI:10.1523/JNEUROSCI.2278-12.2012

Copyright $\odot 2012$ the authors $\quad 0270-6474 / 12 / 3216031-09 \$ 15.00 / 0$
}

The primary purpose of this study was to test for a causal involvement of the AIC in decision making under risk by using reversible pharmacological lesions. We investigated the effect of temporarily inactivating rat AIC on risk preference in two different gambling tasks, one with risky reward amounts, the other with risky reward delays. We aimed to establish the generality of the inactivation effects on risk preference beyond a single task. We also used a control task to dissociate the main findings from effects on other properties of reward, such as reward delay and magnitude.

We contrasted AIC inactivations with inactivations of the orbitofrontal cortex (OFC) within the same subjects. Patients with damage to the ventromedial prefrontal area including the OFC make abnormal risky decisions in the Iowa Gambling task (Bechara et al., 1994). In human neuroimaging, the OFC activates in various gambling tasks (Rogers et al., 1999b; Ernst et al., 2002; Fukui et al., 2005; Tobler et al., 2007; Li et al., 2010). In rats, OFC-lesions increased preference for risk (Pais-Vieira et al., 2007). Based on these studies, the OFC has been thought to play a crucial role for optimal decision making under risk. Our second purpose was to test the generality of this role in two distinct risk tasks, one in which risk arises in reward amount and one in which it arises in reward delay. Third, we aimed to clarify the functional differences between adjacent AIC and OFC in risky decision making by using the same experimental procedure and subjects. Such a within-subject design with reversible inactivations and washout periods allowed us to tightly control for individual behavioral differences and minimize compensatory effects resulting from lesions.

\section{Materials and Methods}

Animals. Twenty-four male Wistar rats weighing 200-320 g were used for the experiments. Rats were individually housed under $12 \mathrm{~h}$ light/dark cycles with light onset at 8:00 P.M. Training and testing took place during the dark phase. Rats were given ad libitum access to food for the duration 
A

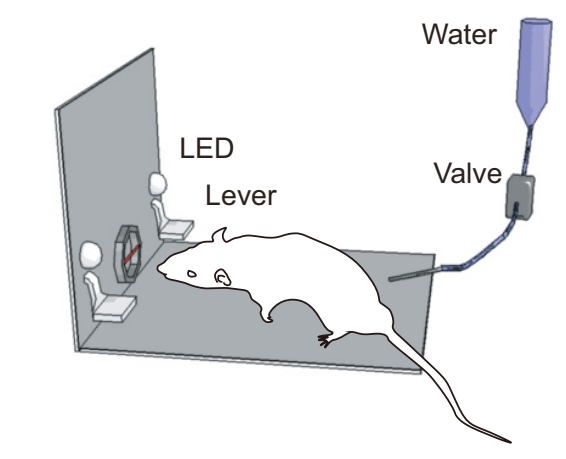

C

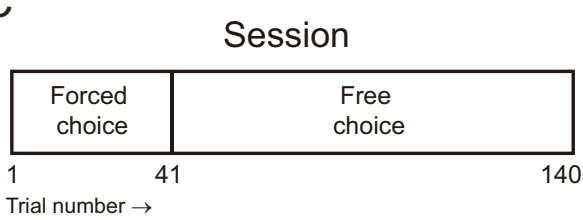

B Amount gambling task

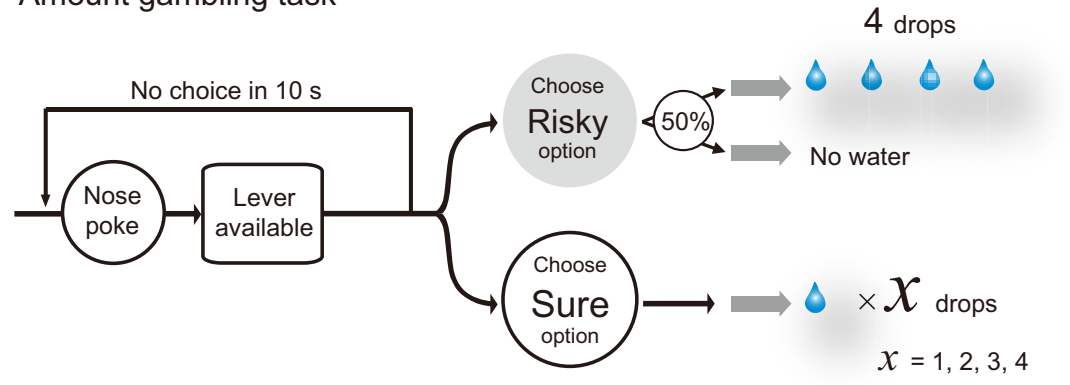

Delay gambling task

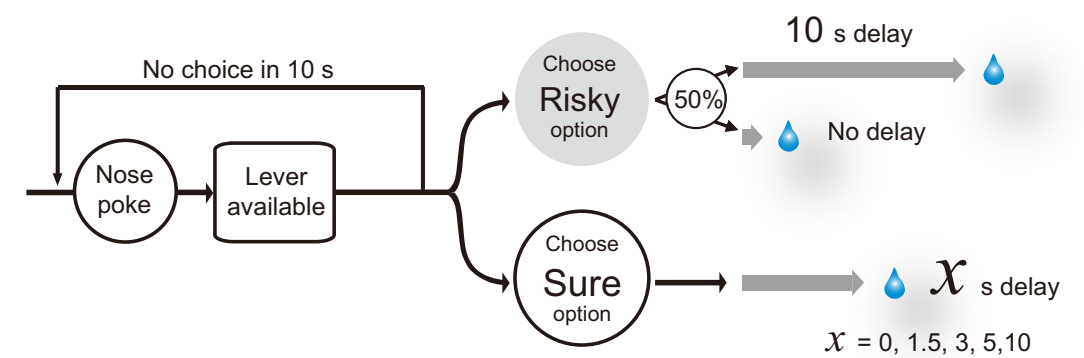

Figure 1. Task design. $A$, Experimental apparatus. $B$, Sequence of events in a trial of the amount gambling task (top) and delay gambling task (bottom). The rats were required to nose-poke to initiate a trial, and then to press a lever. Water was given as reward. Both tasks required the rats to choose between a risky option and a sure option. $C$, Session structure. In the first 40 forced trials, the rats learned the relationship between the lever positions and the outcomes. In the following 100 free choice trials, the rats were free to choose between the two options.

of the experiments but limited access to drinking water because water was used as reward for the experiment. To prevent weight loss, rats were given additional water after the daily experiment finished. Their body weights were monitored daily. Throughout the experiments, animals were treated in accordance with the National Institutes of Health Guide for the Care and Use of Laboratory Animals and Tohoku University Guidelines for Animal Care and Use.

Overall structure of the experiment. We investigated the effects of AIC and OFC inactivation on rats' decision making in two types of gambling tasks (the amount and delay gambling task) and intertemporal choice task (as a control). The basic task for the rat was to get water by choosing one of two levers that were associated with different outcomes. The rats learned the basic rules of the tasks in training 1 , and were trained to discriminate the difference in reward amount or delay in training 2 (for details, see below). Then, we investigated behavioral performances without drug injections in both gambling tasks and in the intertemporal choice task. Finally, we implanted guide cannulae for drug injections and, after a recovery period, conducted pharmacological tests in those same tasks.

Apparatus. Experiments were conducted in dimly lit sound-attenuated boxes $(60 \times 45 \times 35 \mathrm{~cm})$. On one wall of the box, a nose-poke hole was located in the center and two levers on each side of the hole. Nose-poke responses were detected by a horizontal infrared beam (OMRON). White LEDs were positioned above each lever. A nozzle delivering water was on the opposite wall. Auditory stimuli were generated by a speaker located in the ceiling. Each device was connected to a computer via a Digital I/O card (PCI-7248; ADLINK Technology) and controlled by an in-house software program (based on $\mathrm{C}++$ ). The boxes were equipped with a fan for ventilation and masking noise. For details of apparatus settings, see Figure $1 A$.

Training 1: Nose-poke and lever press. Training 1 consisted of three steps. First, rats were trained to press either lever to get water. An auditory stimulus $(2 \mathrm{kHz}, 1 \mathrm{~s})$ was presented upon lever press and water delivery followed. Second, rats were trained to press the lever when a visual stimulus was presented. The visual stimulus also indicated which lever the rats should press. If the left visual stimulus was presented, the rats were required to press the left lever (correct case). They could not get water if they pressed the right lever (wrong case) and were required to press the left lever again in the next trial. When rats pressed the wrong lever, the auditory stimulus was not presented. Once a lever was pressed, visual stimuli disappeared and levers were unavailable until the next trial. Finally, rats were trained to nose-poke into the central hole to start a trial. They subsequently pressed one of the available levers and got water from the nozzle at the opposite wall of the box. If rats did not press any lever within $10 \mathrm{~s}$ after nose-poke, the trial was cancelled and visual stimuli disappeared until the next trial.

Training 2: Amount and delay discrimination task. Before they were tested in the amount, delay, or intertemporal choice tasks, rats were trained to discriminate differences in either amount or time. The amount discrimination task consisted of a choice between four and two drops of water, the delay discrimination task consisted of a choice between no delay and $1.5 \mathrm{~s}$ delay. In both discrimination tasks, a session consisted of 40 forced choices for learning the relationship between levers and outcomes and 100 free choices for measuring rats' ability to discriminate. In the first 40 forced-choice trials, available levers were limited to one on each trial. Rats experienced the two options equally often. In the following 100 free-choice trials, both levers were available, and rats could choose between the two options. Assignment of outcomes to levers was counterbalanced between sessions. Sessions were conducted up to three times a day and lasted $>3 \mathrm{~h}$. A rat was moved to the next step (gambling task) when it had performed 10 or more consecutive sessions in which they chose the better option $>65 \%$ of the time. In both discrimination tasks, rats chose the better option in $>85 \%$ of the time in most sessions.

Trial structure. In both discrimination tasks, a $1 \mathrm{~s}$ period was provided after the lever press as moving time; that is, water was delivered at least $1 \mathrm{~s}$ after the lever press. The duration of delay was implemented after this $1 \mathrm{~s}$ moving time. The auditory stimulus was presented during lever press when water was given. In the amount discrimination task, one drop of water was $50 \mu \mathrm{l}$, and the interval between each drop was $700 \mathrm{~ms}$. Duration of the delay was $3 \mathrm{~s}$. In the delay discrimination task, rats were given $100 \mu \mathrm{l}$ of water per trial.

Depending on the experimental task in which they later were used, rats were tested in the amount, delay, or both discrimination tasks. Thus, the nine $(10,5)$ rats that were used in the amount gambling (delay gambling, 
intertemporal choice) task had been tested for their amount (delay, amount and delay) discrimination ability in the amount (delay, both) discrimination task.

Amount and delay gambling tasks. Risk can arise from variability in reward amount or delay (Logan, 1965; Kacelnik and Bateson, 1996). In the present study, we investigated the effects of AIC or OFC inactivation on risky decision making associated with reward amount and delay using two gambling tasks (the amount gambling task and the delay gambling task), which were modified from previously described procedures (Logan, 1965). In both tasks, water-deprived rats were required to choose and press one of two levers for water as reward (Fig. $1 A, B$ ). In the amount gambling task, rats were required to choose between a risky option (variable amount: 4 drops or no water, 50/50 chance) and a sure option (fixed amount: $x$ drops of water; $x=1,2,3,4 ; x$ was fixed in a session). In the delay gambling task, rats were required to choose between a risky option (variable delay until reward delivery: 0 or $10 \mathrm{~s}, 50 / 50$ chance) and a sure option (fixed delay: $x=0,1.5,3,5,10 \mathrm{~s}, x$ was fixed in a session; Fig. $1 B$ ). The overall task designs and procedures in the two gambling tasks were the same as in the amount and delay discrimination tasks.

Before we conducted pharmacological experiments, we investigated behavioral performance without drug injections at each $x$ in both gambling tasks. More than 12 sessions were conducted at each level of $x$. Percent choice of the risky option in each individual was the average of 10 sessions selected from the last consecutive 12 sessions, eliminating the two sessions with maximum and minimum percent choice of the risky option. Subjective equivalence points between risky and sure options (percent choice of the risky option $=50 \%$ ) were calculated based on logistic sigmoid functions that were fitted to the observed value using the least-square method.

Intertemporal choice task. If inactivation of either the AIC or OFC disrupted the recognition of the difference in reward amount or delay, or affected value processing under sure conditions, percent choice of the risky option could have been affected in the amount and delay gambling tasks. To test this possibility, we investigated the effect of AIC or OFC inactivation in an intertemporal choice task. In this task, rats were required to choose between a larger later option and a smaller sooner option. This task has been used previously in delay discounting studies (Cardinal et al., 2001; Kalenscher and Pennartz, 2008), and our experimental design was modified from the one described by Evenden and Ryan (1996). If AIC or OFC inactivation affects either discrimination or evaluation of reward amount or delay, percent choice of the two options would change in this task. The larger later option provided four drops of water after a delay of $x$ seconds ( $x=0,3,5,7,10 ; x$ was fixed in a session). The smaller sooner option provided two drops of water immediately. The overall task design and experimental procedures were the same as in the amount and delay gambling tasks. We calculated the subjective equivalence point between larger later and smaller sooner options by using the same methods as in the two gambling tasks.

Surgery. After the rats were tested for their behavioral performances in the amount gambling task, delay gambling task, or intertemporal choice task, they were surgically implanted with stainless steel guide cannulae (0.6 mm diameter) targeting $1 \mathrm{~mm}$ above bilateral AIC (AP +3.0, ML $\pm 4.2, \mathrm{DV}-5.6 \mathrm{~mm})$ and $\mathrm{OFC}(\mathrm{AP}+4.4, \mathrm{ML} \pm 2.2, \mathrm{DV}-5.0 \mathrm{~mm})$. Under ketamine $(80.0 \mathrm{mg} / \mathrm{kg})$ and xylazine $(0.8 \mathrm{mg} / \mathrm{kg})$ anesthesia, the scalp was retracted, and craniotomies were made bilaterally above the target sites of the cannulae. Four guide cannulae $(0.6 \mathrm{~mm}$ diameter $)$ were inserted and fixed with dental cement. To prevent clogging by blood clots, dummy injection cannulae $(0.3 \mathrm{~mm}$ diameter $)$ were inserted into the guide cannulae. Rats were given 1 week of recovery after the surgery. After the recovery period, they were retrained in either the amount or delay discrimination task in a week. In the retraining period, rats were handled $30 \mathrm{~min}$ before each session to get them acclimatized to the procedure of drug injection.

Drugs. Either the AIC or OFC was inactivated by injecting a mixture of muscimol (GABA A receptor agonist) and baclofen (GABA B receptor agonist) (Sigma), which have been used in previous inactivation studies (Ghods-Sharifi et al., 2009; Takahashi et al., 2009). Muscimol and baclofen were dissolved separately in physiological saline at a concentra- tion of $200 \mathrm{ng} / \mu \mathrm{l}$, and combined in equal volumes. The final concentration of each drug was $100 \mathrm{ng} / \mu \mathrm{l}$. The concentration of drugs was determined by referring to previous studies (Ghods-Sharifi et al., 2009; Horst and Laubach, 2009; Takahashi et al., 2009) and our preliminary experiment. The volume of drugs was $100 \mathrm{nl}$ per hemisphere. We used lower drug volumes than in the previous studies to prevent diffusion between AIC and OFC, which adjoin each other. To test the extent of drug diffusion, we conducted additional tests in which two types of dye were injected into the AIC and OFC, using the same injection procedures as the main experiment. As smaller molecules are generally more likely to diffuse (Hayduk and Laudie, 1974), we used thionine and pontamine sky blue, whose molecular weight was relatively close to and larger than that of the drugs, respectively (muscimol: 114.1, baclofen: 213.66, thionine: 263.75, pontamine sky blue: 992.8). Despite the difference in molecular weight, the extents of diffusion of the two dyes were both $1.5 \mathrm{~mm}$ in width. Edeline et al. (2002) report a similar extent of diffusion after the same amount of muscimol injection into the cortex. Importantly, there was no overlap between injection sites.

Injection procedure. Drug or saline was injected $30 \mathrm{~min}$ before the behavioral tests. During the drug injection, rats were handled on the experimenter's lap without anesthesia. The dummy cannula was removed from the implanted guide cannula, and the injection cannula was inserted into the target region via the guide cannula. The injection cannulae were stainless steel needles $0.3 \mathrm{~mm}$ in diameter and extending 1 $\mathrm{mm}$ from the tip of the guide cannulae. Injection cannulae were connected to a microliter Hamilton syringe by $0.26-\mathrm{mm}$-diameter polyethylene tubing. The drug or saline was delivered at a rate of $50 \mathrm{nl} / \mathrm{min}$ for 2 min (100 nl per hemisphere) under syringe infusion pump control. Injection cannulae were left in place for $1 \mathrm{~min}$ after injection to allow diffusion of the fluid. Injections to each hemisphere were performed separately; the entire injection procedure took 7-10 min. Finally, we checked for clogging of the injection cannula after the injection. Data were excluded from the analysis if the injection cannula were clogged. To habituate the rats to the injection procedure, they underwent a mock injection during preinjection sessions (the last 5 sessions before injection conditions).

Pharmacological test schedule. Each rat underwent four injection conditions (AIC/drugs, AIC/saline, OFC/drugs, and OFC/saline in a randomized order). All injections were spaced at least $2 \mathrm{~d}$ apart. We treated the last five preinjection sessions as a baseline. The averages of the standard deviations of the risky choices in the last five preinjection sessions were $11.1 \%$ in the amount gambling task, $10.1 \%$ in the delay gambling task, and $10.4 \%$ in the intertemporal choice task.

Histology. After experiments were completed, the brains were sliced into $50 \mu \mathrm{m}$ coronal sections and stained with thionine. Slices were examined under a microscope to identify locations of cannulae. Cannulae placements were classified using the rat brain atlas (Paxinos and Watson, 2007).

Data analysis. Statistical analysis was conducted using SPSS and Microsoft Excel. In both the amount and the delay gambling task, four behavioral parameters were measured for each animal: percent choice of the risky option in 100 free choices, correct rate in 40 forced choices, average reaction time in 100 free choices, and number of reaction omissions in the whole session.

The population's percent choice of the risky option was the average percent choice of the risky option of all individuals. Comparisons between percent choice of the risky option and chance level (50\%, random choice between the two options) were made by one-sample $t$ test (significance level: $p<0.05$ ). To calculate the subjective equivalence point between the risky and sure options (percent choice of the risky option $=$ $50 \%)$, a logistic sigmoid function $(f(x)=a+b /(1+\exp (-(x-c) / d)$; $a, b, c$, and $d$ were free parameters) was fitted to the observed choice frequencies using the least-squares method.

For the analysis of the injection conditions, first we compared the percent choices of the risky option between the pharmacological treatment session (postinjection) with the average of the last five sessions before injection (preinjection), and one session conducted on the day after postinjection (recovery). Comparisons of percent choice of the risky option between preinjection, postinjection, and recovery were made using a 
one-way ANOVA. If the outcome of this ANOVA yielded significant effects at the $p<0.05$ level, a post hoc $t$ test with Bonferroni correction was performed (significance level: $p<0.05$, Bonferroni-corrected). The changes in the percent of risky option choice from preinjection to postinjection (the percent of risky option in postinjection minus that of preinjection) were compared between the drug and saline injection conditions on an individual animal basis by paired $t$ test (significance level: $p=0.05$ ).

Comparisons of the correct rate between drugs and saline injection were made by nonparametric Wilcoxon signed ranks test (significant level: $p<0.05)$. A nonparametric test was used because the correct rate was extremely high and not normally distributed. Comparisons of reaction time and reaction omission between the drugs and saline injection were made by paired $t$ tests (significant level: $p<0.05$ ).

In the intertemporal choice task, data collection and analysis was performed as in the two gambling tasks. Comparisons of percent choice of the larger later option between preinjection, postinjection, and recovery sessions were made using one-way ANOVA.

Data were excluded from the analysis when the injection cannulae were found to be clogged or when the tip of the injection cannulae deviated from the target region with histological investigation (Fig. 3B, $\mathrm{x}$ symbol). The final numbers for each injection condition were nine for every experiment in the amount gambling task and nine (AIC/drugs), six (AIC/saline), seven (OFC/drugs), and six (OFC/saline) in the delay gambling task. Note that the results remained significant when all data were included.

\section{Results}

\section{Behavior in two gambling tasks}

Figure 2, $A$ and $B$, show behavioral performance in the amount $(N=9)$ and delay $(N=10)$ gambling tasks, respectively. We focus on two aspects of these results here. One aspect is whether the rats understood the difference between risky and sure options. In both tasks, percent choice of the risky option changed gradually (not abruptly) depending on the value of the sure option. This result suggests that the rats were attracted not only to either outcome of the risky and sure options, but compared the three outcomes provided by the two options (i.e., they were sensitive to changes in the sure amount).

Next, we asked which option the rats preferred when the risky and sure option had the same expected value in the two gambling tasks. Risky and sure options had the same expected value at $x=$ 2 in the amount gambling task and at $x=5$ in the delay gambling task. Because each option, on average, provides the same reward amount or delay at these conditions, rats with no preference for either option would choose the risky option in $\sim 50 \%$ of the trials. In the amount gambling task, six of nine rats exhibited a significant preference for the risky option at $x=2$ (risk-seeking type), and the other three rats had no significant preference (riskindifferent type). In the population, the percent choice of the risky option was significantly higher than chance level (60.5 \pm $\left.3.1 \%, t_{(9)}=7.67, p<0.05\right)$. In the delay gambling task, all 10 rats exhibited a preference for the risky option at $x=5(84.1 \pm 2.1 \%$, $\left.t_{(10)}=16.14, p<0.05\right)$. These behavioral tendencies in both tasks are consistent with previous reports that in general, animals exhibit various preferences for risk in reward amount and prefer risk in reward delay when risky and sure options have the same expected value (Kacelnik and Bateson, 1996; Weber et al., 2004).

To allow risk preference to change by inactivations, we investigated the $x$ value at which risky and sure options were equivalent for the rats (percent choice of the risky option $=50 \%$, subjective equivalence point) by drawing sigmoid curves based on logistic functions that were fitted to the observed value $\left(r^{2}>\right.$ 0.99 in both tasks). The subjective equivalence point between the risky and sure option was 2.4 in the amount gambling task and 2.3 in the delay gambling task. In the following experiments, the

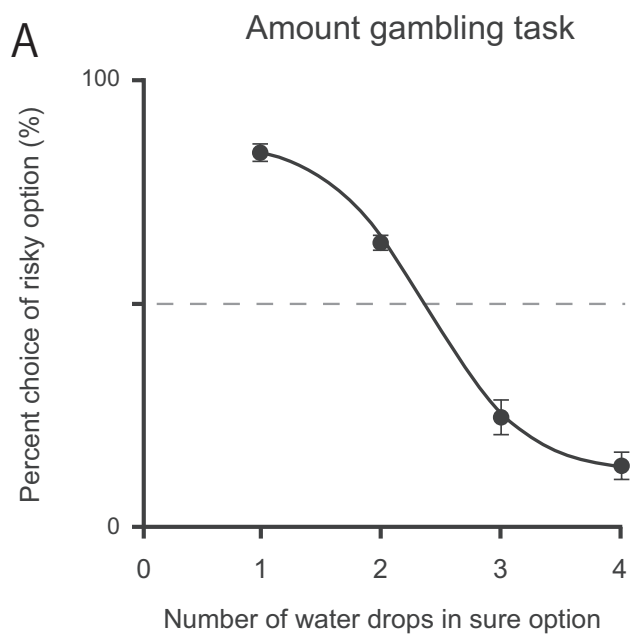

B Delay gambling task

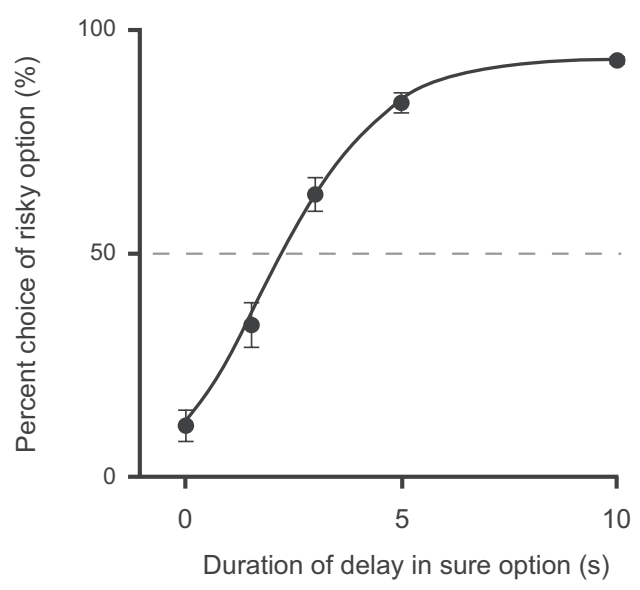

Figure 2. Percent choice of the risky option in the amount $(\boldsymbol{A}, N=9)$ and delay $(\boldsymbol{B}, N=10)$ gambling tasks. Sigmoid curves were drawn based on logistic functions that were fitted to observed values $\left(r^{2}>0.99\right.$ in both tasks). When the risky and sure options had the same expected value ( $x=2$ in the amount gambling task, $x=5$ in the delay gambling task), rats significantly preferred the risky option ( $p<0.05$, both tasks). Calculated from sigmoid logistic functions, the subjective equivalence points between risky and sure options were 2.4 in the amount gambling task and 2.3 in the delay gambling task. Error bars indicate SEM.

rats were tested for risk preference at $x=2$ in the amount gambling task because this was the nearest integer number to the subjective equivalence point, and at their respective subjective equivalence point in the delay gambling task because delays could be adjusted precisely to individual subjective equivalence points.

\section{Inactivation of AIC and OFC in gambling tasks}

The rats were implanted with cannulae for local microinjection of drugs. Figure 3 shows guide and injection cannulae tracks and injection sites. In Figure 4, $A$ and $B$, the effects of inactivation of the AIC and OFC on risk preference are shown as group data. Changes in percent choice of the risky option between preinjection, postinjection, and recovery were detected with a one-way ANOVA and further specified by post hoc $t$ tests with Bonferroni correction. Saline injection into either the AIC or OFC did not affect risk preference in either gambling task (amount gambling task: $\mathrm{AIC}, p=0.69$; OFC, $p=0.66$; delay gambling task: $\mathrm{AIC}, p=$ 0.66 ; OFC, $p=0.86$; one-way ANOVA). In contrast, inactivation by muscimol and baclofen injection changed risk preferences. The direction of change was similar for both tasks but different 
A
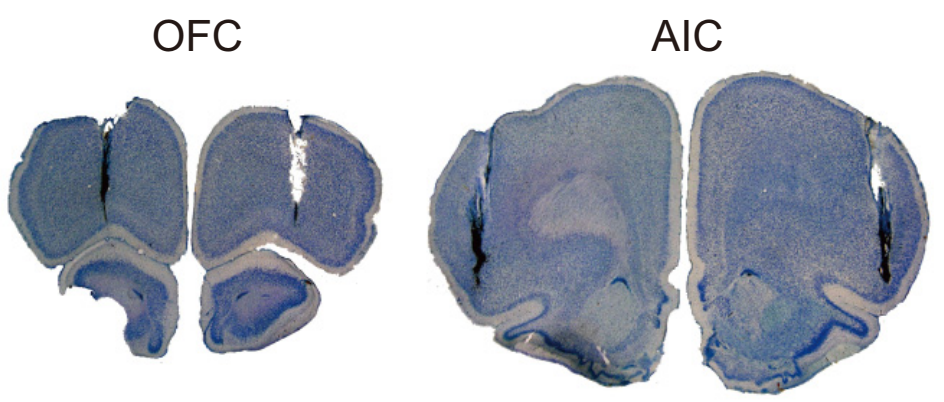

$\mathrm{B}$
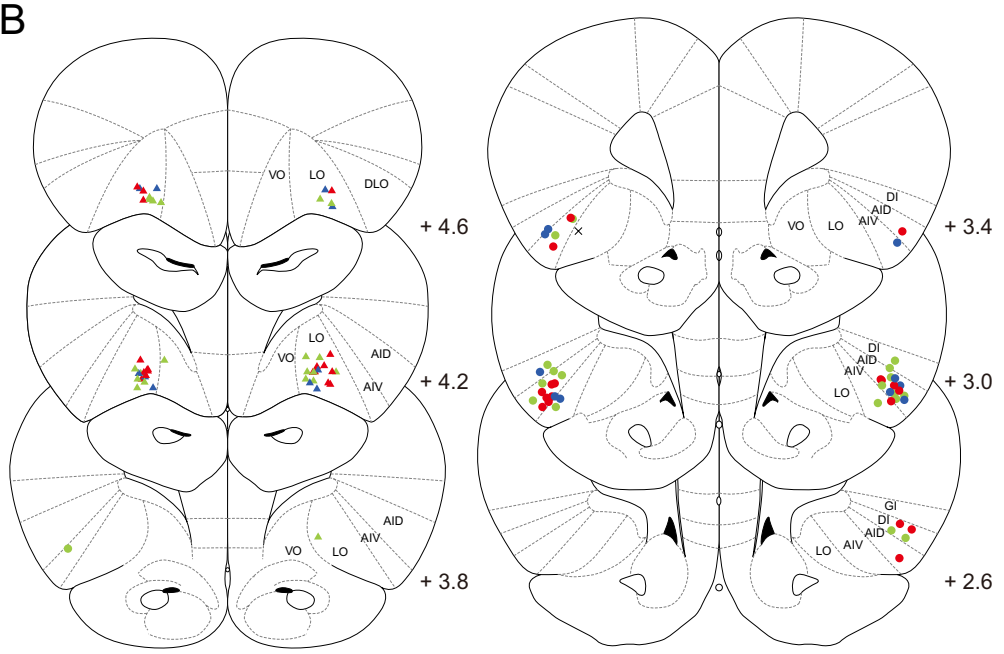

$\triangle:$ OFC

$\mathrm{O}:$ AlC

$X$ : Out of traget region
Red: Amount gambling task Green: Delay gambling task Blue: Intertemporal choice task

Figure 3. Locations of injection. Rats were bilaterally implanted with $0.6-\mathrm{mm}$-diameter guide cannulae through which 0.3$\mathrm{mm}$-diameter injection cannulae were inserted. $\boldsymbol{A}$, Typical tracks of guide and injection cannulae. $\boldsymbol{B}$, Locations of tip of injection cannulae in the AIC and OFC. Plates are adaptations from the atlas of the rat brain (Paxinos and Watson, 2007). AID, Dorsal agranular insular cortex; AIV, ventral agranular insular cortex; DI, dysgranular insular cortex; DL0, dorsolateral orbitofrontal cortex; $\mathrm{Gl}$, granular insular cortex; LO, lateral orbitofrontal cortex; VO, ventral orbitofrontal cortex.

depending on injection site. Inactivation of the AIC decreased risk preference whereas inactivation of the OFC increased it (oneway ANOVA, all $p<0.05$; post hoc $t$ test with preinjection vs postinjection at each drug injection condition, Bonferronicorrected, all $p<0.05)$. There were no differences in inactivation effects on risk preference between risk-seeking and risk-indifferent rats in the amount gambling task.

We also analyzed the changes in risk preference on the singlesubject level. Figure $4 C$ shows the individual changes in percent choice of the risky option from preinjection to postinjection (i.e., percent choice of the risky option postinjection minus that of preinjection). Risk preference changes induced by drug injection differed significantly from saline injection (all $p<0.05$, paired $t$ test with drugs vs saline).Thus, AIC or OFC inactivation also had distinct effects on risk taking at the single-subject level, regardless of whether risk was in amount or delay.

To test whether the risk preferences developed over the 100 free-choice trials, we compared the percent choices of the risky option for each injection condition by dividing the free-choice trials into four blocks. There were no significant differences in risky choices between blocks [all $p>0.1$, repeated-measures twoway ANOVA (drugs/saline $\times$ blocks)], and interactions between drugs/saline and blocks were not significant either (however, the $p$ value of the interaction between drugs/saline and blocks in OFC

injections in the amount gambling task was 0.06 ). Thus, there was no significant development of the risk preference during a session.

\section{Control analysis: less specific effects of AIC or OFC inactivation?}

Prefrontal cortex is thought to be involved in cognition, motor performance, and attention (Fuster, 2008). We therefore investigated whether AIC and OFC inactivation affected performance in domains other than risk taking. More specifically, we compared three behavioral parameters after muscimol and baclofen injection versus saline injection: correct rate in forced choices (cognition), reaction time (duration from nose-poke to lever press) in free choices (motor performance), and number of reaction omissions (no lever press in $10 \mathrm{~s}$ after nose-poke) in the whole session (attention) (Table 1).

Forced choice required pressing a specified lever to get a reward, but rats could also press a nonspecified lever (for no reward). The correct rate in forced choices could have been affected by various cognitive dysfunctions, but AIC and OFC inactivation had no significant effect in either the amount gambling task (AIC: $z=-1.41, p=0.16$, OFC: $z=-1.0, p=0.32$, Wilcoxon signed ranks test) or the delay gambling task (AIC: $z=0$, $p=1.0$, OFC: $z=0, p=1.0$, Wilcoxon signed ranks test).

Although there was a possibility that muscimol and baclofen injection might affect motor performance by leaking to the secondary motor area through the guide cannulae, there were no significant differences in reaction time between saline and drug injection into either the AIC or OFC in the amount gambling task (AIC: $p=0.07$, OFC: $p=0.59$, paired $t$ test) or the delay gambling task (AIC: $p=0.15$, OFC: $p=0.75$, paired $t$ test).

Reaction omission could result from a deficit in attention. However, reaction omissions rarely happened in any of the 140 trials even with drug injections. There were no significant differences in the number of reaction omissions between saline and drug injection into either the AIC or OFC in either the amount gambling task (AIC: $p=0.07$ OFC: $p=0.14$, paired $t$ test) or the delay gambling task (AIC: $p=0.22$, OFC: $p=0.20$, paired $t$ test).

\section{Control experiment: intertemporal choice task}

To test whether inactivation of the AIC and OFC affected the recognition of the difference in reward amount or delay, or value processing under sure conditions, we investigated the effect of AIC and OFC inactivation in the intertemporal choice task. Figure $5 A$ shows the behavioral performance in the intertemporal choice task $(N=5)$. In the same way as for the two gambling tasks, we calculated the subjective equivalence point between larger later and smaller sooner options for each individual $(x=$ 5-7), and conducted pharmacological experiments at these points. Figure $5 B$ shows that there were no significant effects on intertemporal choice by AIC and OFC inactivation $(p=0.56$ and 
A

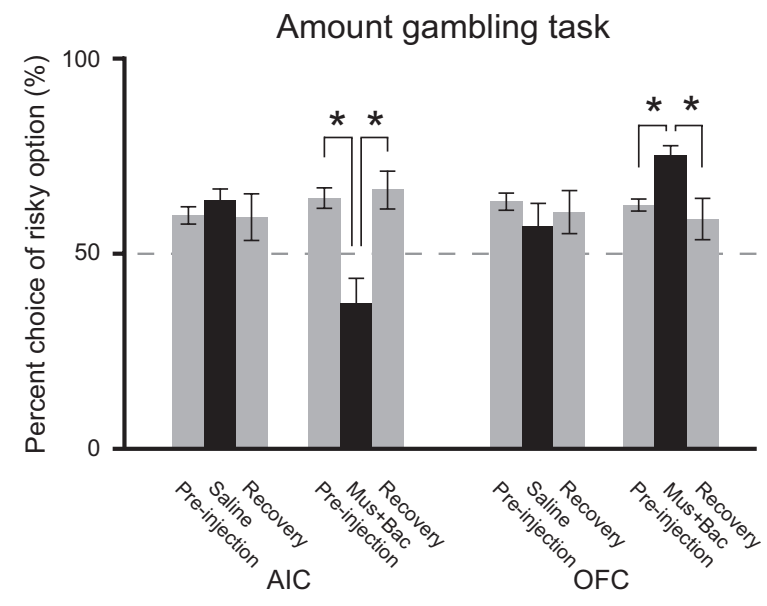

$\mathrm{B}$

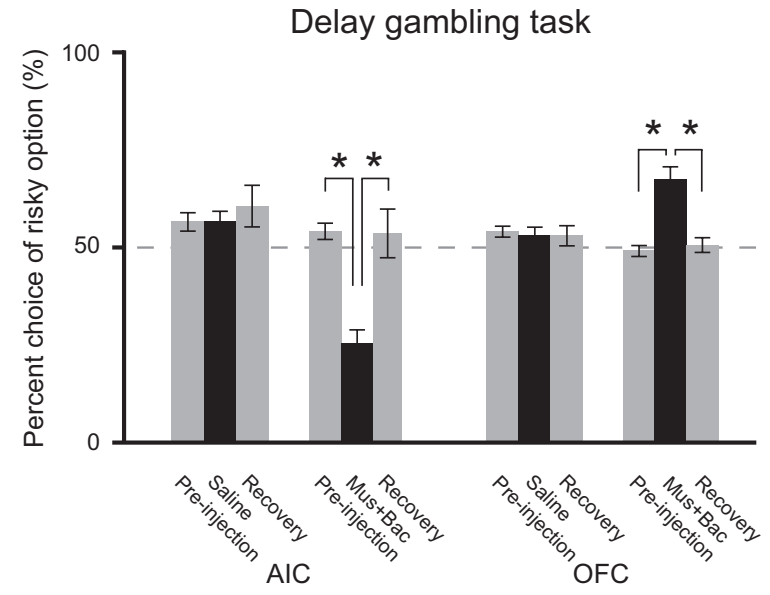

C

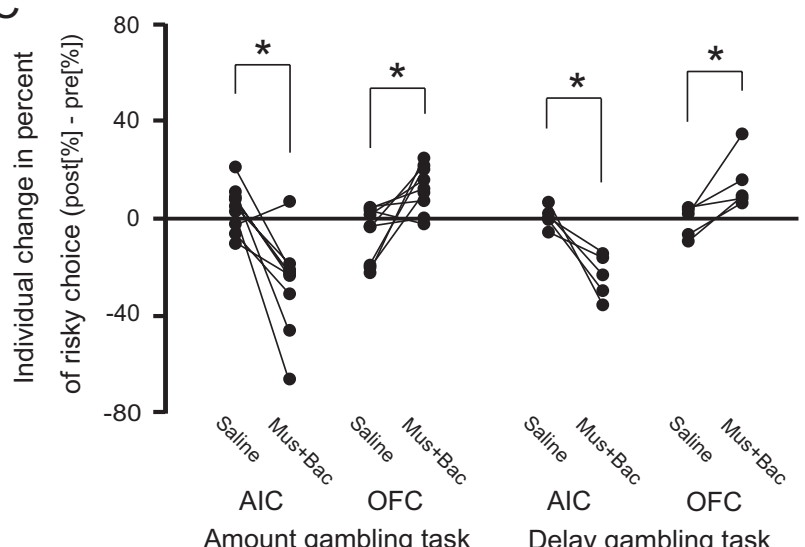

Mus: muscimol Bac: baclofen

Figure 4. Effects of AIC and OFC inactivation on risk preference in the two gambling tasks. In both the amount $(\boldsymbol{A})$ and delay $(\boldsymbol{B})$ gambling task, AIC inactivation significantly decreased risk preference. In contrast, $0 \mathrm{FC}$ inactivation significantly increased risk preference. Comparisons were made by ANOVA and post hoc $t$ test. * Significant difference between all combinations by post hoc $t$ test $(p<0.05$, Bonferroni-corrected). C, individual changes in risky choices. Drugs and saline injection data belonging to the same individual are connected with a straight line. Risk preferences after drug injection significantly differed from those after saline injection (*all $p<0.05$, paired $t$ test). Error bars indicate SEM.

$p=0.81$, respectively, ANOVA $)$ and by saline injection $(p=0.56$ and $p=0.51$, respectively, ANOVA).

It is possible that inactivations affected the value processing of amount and delay in opposite ways such that they canceled out. If so, AIC and OFC inactivations would affect the discrimination performances in the amount and delay discrimination tasks. However, our initial preliminary experiments confirmed that AIC and OFC inactivations have no effect on discrimination performance in each discrimination task, so this possibility seems unlikely.

\section{Discussion}

Previous studies have found various neural correlates of decision making under risk, such as the amygdala, nucleus accumbens, cingulate cortex, dorsolateral prefrontal cortex, ventromedial prefrontal cortex, orbitofrontal cortex, and insular cortex (McCoy and Platt, 2005; Tom et al., 2007; Venkatraman et al., 2009; Mohr et al., 2010). In the present study, we focused on the causal roles of two limbic regions, AIC and OFC. In two different types of gambling tasks, we found that inactivation of the AIC decreased risk preference whereas inactivation of the OFC increased risk preference. Because the effects of these inactivations were similar for both tasks, inactivations appear to have affected risk processing generally and directly rather than affecting more specific reward-amount and delay-value processing or having indirect effects. This interpretation is supported by the result of the control experiment suggesting that AIC and OFC inactivations had no significant effects on intertemporal choice. The AIC and OFC seem to be important for decision making under risky conditions and not always necessary for value-based decision making under sure conditions. In addition, AIC and OFC inactivations had no significant effects on less specific factors of task performance, such as correct rate, reaction time, or reaction omission. Based on these results, we propose that inactivation of either AIC or OFC primarily changed risk preference. Most importantly, the change occurred in opposing directions for the two regions.

\section{Intact AIC promotes risk taking}

Previous human imaging studies have reported AIC activation in risky decision making (Paulus et al., 2003; Kuhnen and Knutson, 2005; Preuschoff et al., 2008; Xue et al., 2010; Burke and Tobler, 2011). Here, we demonstrated a causal involvement of the AIC in risky decision making, and, by extension, suggest that the intact AIC normally promotes risk taking. This idea concurs with previous findings that insular activation was stronger when participants took risky versus sure decisions (Paulus et al., 2003) and that high insula activation was more likely to occur when participants took a risky decision after taking a no-risk decision in the previous trial (Xue et al., 2010).

The findings of AIC involvement in drug addiction are consistent with our results of AIC inactivation. Damage to the insula disrupted addiction to cigarette smoking (Naqvi et al., 2007). Insula inactivation prevented amphetamine-seeking in a place-preference task (Contreras et al., 2007). From these observations, the AIC is thought to induce an urge for the pleasurable interoceptive effects of drug taking (Naqvi et al., 2007). However, drugs are risky substances, often leading not only to pleasurable but adverse effects. Together with our findings, the AIC may promote risk seeking in situations in which both positive and negative outcomes can ensue. More generally, the AIC has been implicated as a key structure in linking multisensory information, affective processing, and previous experiences through connections with the posterior insular cortex, the amygdala, and the hippocampal system (Shi and Cassell, 1998; Delatour and Witter, 2002; Reynolds and Zahm, 2005; Craig, 2009). Given these interactions, the AIC may process risk information by using interoceptive and emotional information, and may promote seeking the better outcome of a risky decision. 
Table 1. No effect on other behaviors by either AIC or OFC inactivation

\begin{tabular}{|c|c|c|c|c|c|c|}
\hline & \multicolumn{3}{|l|}{ AIC } & \multicolumn{3}{|l|}{ OFC } \\
\hline & Saline & Mus + Bac & & Saline & Mus $+\mathrm{Bac}$ & \\
\hline \multicolumn{7}{|l|}{ Amount gambling task } \\
\hline Correct rate in 40 forced choices (\%) & $100 \pm 0$ & $99.8 \pm 0.1$ & ns & $100 \pm 0$ & $990.9 \pm 0.1$ & ns \\
\hline Reaction time in 100 free choices (ms) & $1260 \pm 274$ & $1560 \pm 125$ & ns & $1116 \pm 156$ & $1174 \pm 91$ & ns \\
\hline Number of reaction omission in whole session & $0.56 \pm 0.18$ & $1.56 \pm 0.50$ & ns & $0.44 \pm 0.18$ & $1.00 \pm 0.29$ & ns \\
\hline \multicolumn{7}{|l|}{ Delay gambling task } \\
\hline Correct rate in 40 forced choices (\%) & $100 \pm 0$ & $100 \pm 0$ & ns & $100 \pm 0$ & $100 \pm 0$ & ns \\
\hline Reaction time in 100 free choices (ms) & $1027 \pm 63$ & $1356 \pm 190$ & ns & $1019 \pm 48$ & $1001 \pm 86$ & ns \\
\hline Number of reaction omission in whole session & $0.33 \pm 0.21$ & $1.42 \pm 0.48$ & ns & $1.17 \pm 0.48$ & $0.67 \pm 0.33$ & ns \\
\hline
\end{tabular}

The effects of AIC or OFC inactivation on three behavioral parameters: correct rate in 40 forced choices, reaction time in 100 free choices, and number of reaction omissions in the whole session. There were no significant differences between inactivations and saline in both gambling tasks, suggesting that inactivations affected performance in risk taking and not other domains. Values are mean \pm SEM. Mus + Bac, muscimol + baclofen; ns, not significant.

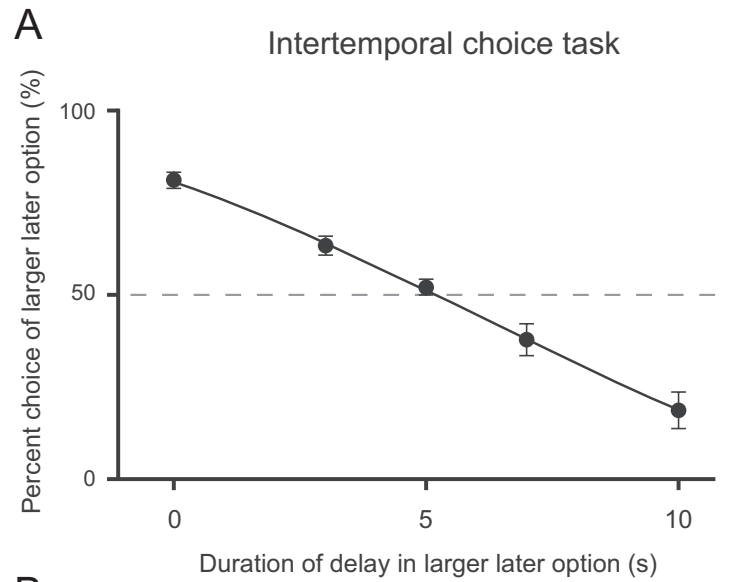

B

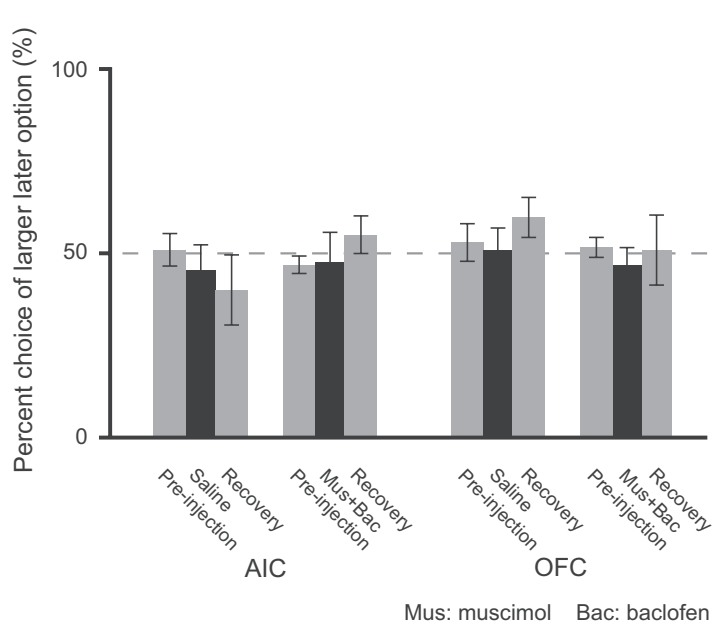

Figure 5. Intertemporal choice task $(N=5)$. $\boldsymbol{A}$, Performance at each $x$ condition and fitted sigmoid curves based on logistic function $\left(r^{2}>0.99\right)$. $\boldsymbol{B}$, Effects of AIC or OFC inactivation on intertemporal choice at subjective equivalence point with larger later and smaller sooner options $(x=5)$. Neither AIC or OFC inactivation had significant effects in this task ( $p=0.56, p=$ 0.81 , ANOVA). Saline injection also had no significant effect ( $p=0.56, p=0.51$, ANOVA). Error bars indicate SEM.

The AIC may also suppress avoiding the worse outcome of a risky decision. In our study, risk preference after losing the gamble in the previous trial decreased by AIC inactivation in both gambling tasks (both $p<0.05$, paired $t$ test with preinjection vs postinjection): with AIC inactivation, the rats were more riskaverse, particularly after losing than with saline. This result indicates that the AIC is also involved in adjusting current behavior in the light of adverse outcomes in risky decisions. Our proposal that the AIC promotes risk taking appears to be at odds with a previous idea that the AIC is involved in loss aversion in risky decision making: high AIC activation preceded switching to riskaversive choices in a financial decision making task (Kuhnen and Knutson, 2005). This contradiction may be resolved if the AIC is sensitive to the potentially worse outcome of the risky option and is recruited to suppress risk avoiding.

The AIC may promote risk seeking in synergy with other brain regions. One of these regions is the amygdala. Both the basolateral and the central nucleus receive strong excitatory inputs from the insular cortex (McDonald, 1998). Moreover, temporary inactivation of the basolateral amygdala made animals more riskaverse (Ghods-Sharifi et al., 2009). Thus, it is conceivable that the intact AIC contributes to and combines with amygdala-mediated risk seeking.

\section{Opposite roles of $\mathrm{AIC}$ and OFC in risky decision making}

In this study, we also confirmed that the OFC plays a crucial role in decision making under risk in the two gambling tasks. Consistent with previous reports on patients with OFC damage (Bechara et al., 1994; Rogers et al., 1999a) and OFC-lesioned rodents (Pais-Vieira et al., 2007), inactivation of the OFC increased risk preference in the present study. The common effects in both amount and delay gambling tasks extend the previous literature by showing a general role of the OFC in risk processing.

At first, our finding that OFC inactivation had no effect on intertemporal choice may appear inconsistent with findings of previous studies that rat OFC lesions result in more impulsive choices (Mobini et al., 2002), less impulsive choices (Winstanley et al., 2004), or both (Mar et al., 2011). One reason could be that the time delay setting used for the larger later option in our study (10 s) was much shorter than the ones used in these previous studies $(30-60 \mathrm{~s})$, making the task less sensitive to impulsivity caused by OFC inactivation. Thus, the effect of OFC inactivation on impulsivity might have gone undetected in our study. In other words, our study distinguished the effects of OFC inactivation on risk preference from those on time discounting.

In agreement with the suggested role of the intact OFC in promoting risk aversion, previous studies revealed an involvement of the OFC in risk processing. Single neurons of the primate OFC responded to stimuli predicting risk and these responses increased monotonically with risk (O'Neill and Schultz, 2010). The responses of rat OFC neurons to the outcomes of risky options in free choice reflected individual risk preference (Roitman and Roitman, 2010). The OFC may contribute to loss processing because human lateral OFC was activated by monetary loss (O'Doherty et al., 2001) and activation of lateral OFC covaried with risk and individual risk aversion (Tobler et al., 2007). From these observations, it is reasonable to assume that the loss of OFC 
function interferes with optimal decision making under risk and causes abnormal risk seeking behavior.

Our results provide new insights into not only the role of the AIC but also functional specialization between the two adjacent cortical areas in risky decision making. Anatomically, the AIC and OFC have distinct topographical connectivity with the striatum and the frontal cortex. Concerning striatal connectivity, the AIC mainly projects to ventral and lateral parts of the striatum, whereas the OFC projects more dorsally and medially (Schilman et al., 2008). Concerning frontal connectivity, the AIC has major interconnections with the anterior cingulate, prelimbic, and infralimbic cortex, whereas the OFC is mainly interconnected with the anterior cingulate cortex and has much less interconnection with prelimbic and infralimbic cortex (Hoover and Vertes, 2011). The important point of our results is that AIC and OFC inactivations had opposite effects on risk preference, and this suggests, to our knowledge for the first time, that the AIC and OFC make different contributions to risky decisions. It could be that the AIC promotes exploration for possible gain, whereas the OFC promotes avoidance of possible loss. Moreover, there is a possibility that in the decision phase the AIC and OFC may functionally compete with each other directly or indirectly. Mutually inhibitory interaction between the AIC and OFC could be achieved by anatomical interconnection between them (Van De Werd and Uylings, 2008). Indirect competition could be achieved by opposite influences on other regions that receive projections from both AIC and OFC. One such candidate is the nucleus accumbens (Reynolds and Zahm, 2005), which is also known to be involved in decision making under risk (Kuhnen and Knutson, 2005; Tom et al., 2007). Future combinations of inactivation and electrophysiological studies may help to reveal the functional relationships between the AIC and the OFC.

\section{Conclusion}

We show that AIC inactivation decreases risk preference in the presently used amount and delay gambling tasks. By contrast, OFC inactivation increases risk preference in these tasks. Within the same subjects, we confirm and expand previous findings regarding OFC in risky decision making, and distinguish the role of the AIC from that of the OFC; by implication, the intact AIC promotes risk taking whereas the intact OFC promotes risk aversion. The relative strength of AIC and OFC activities may impact whether we take a risk or avoid it.

\section{References}

Bechara A, Damasio AR, Damasio H, Anderson SW (1994) Insensitivity to future consequences following damage to human prefrontal cortex. Cognition 50:7-15.

Burke CJ, Tobler PN (2011) Reward skewness coding in the insula independent of probability and loss. J Neurophysiol 106:2415-2422.

Cardinal RN, Pennicott DR, Sugathapala CL, Robbins TW, Everitt BJ (2001) Impulsive choice induced in rats by lesions of the nucleus accumbens core. Science 292:2499-2501.

Contreras M, Ceric F, Torrealba F (2007) Inactivation of the interoceptive insula disrupts drug craving and malaise induced by lithium. Science 318:655-658.

Craig AD (2009) How do you feel-now? The anterior insula and human awareness. Nat Rev Neurosci 10:59-70.

Delatour B, Witter MP (2002) Projection from parahippocampal region to the prefrontal cortex in the rat: evidence of multiple pathways. Eur J Neurosci 15:1400-1407.

Doya K (2008) Modulators of decision making. Nat Neurosci 11:410-416.

Edeline JM, Hars B, Hennevin E, Cotillon N (2002) Muscimol diffusion after intracerebral microinjections: a reevaluation based on electrophysiological and autoradiographic quantifications. Neurobiol Learn Mem 78: $100-124$.
Ernst M, Bolla K, Mouratidis M, Contoreggi C, Matochik JA, Kurian V, Cadet JL, Kimes AS, London ED (2002) Decision-making in a risk-taking task: a PET study. Neuropsychopharmacology 26:682-691.

Evenden JL, Ryan CN (1996) The pharmacology of impulsive behavior in rats: the effects on drugs on response choice with varying delays of reinforcement. Psychopharmacology 128:161-170.

Fukui H, Murai T, Fukuyama H, Hayashi T, Hanakawa T (2005) Functional activity related to risk anticipation during performance of the Iowa gambling task. Neuroimage 24:253-259.

Fuster JM (2008) The prefrontal cortex, fourth edition. London: Academic. Ghods-Sharifi S, St Onge JR, Floresco SB (2009) Fundamental contribution by the basolateral amygdala to different forms of decision making. J Neurosci 29:5251-5259.

Hayduk W, Laudie H (1974) Prediction of diffusion-coefficients for nonelectrolytes in dilute aqueous-solutions. Aiche J 20:611-615.

Hoover WB, Vertes RP (2011) Projections of the medial orbital and ventral orbital cortex in the rat. J Comp Neurol 519:3766-3801.

Horst NK, Laubach M (2009) The role of rat dorsomedial prefrontal cortex in spatial working memory. Neuroscience 164:444-456.

Kacelnik A, Bateson M (1996) Risky theories: the effects of variance on foraging decisions. Am Zool 36:402-434.

Kalenscher T, Pennartz CM (2008) Is a bird in the hand worth two in the future? The neuroeconomics of intertemporal decision-making. Prog Neurobiol 84:284-315.

Kuhnen CM, Knutson B (2005) The financial risk taking. Neuron 47:763770.

Li X, Lu ZL, D’Argembeau A, Ng M, Bechara A (2010) The Iowa gambling task in fMRI images. Hum Brain Mapp 31:410-423.

Logan FA (1965) Decision making by rats: uncertain outcome choices. J Comp Physiol Psychol 59:246-251.

Mar AC, Walker AL, Theobald DE, Eagle DM, Robbins TW (2011) Dissociable effects of lesions to orbitofrontal cortex subregions on impulsive choice in the rat. J Neurosci 31:6398-6404.

McCoy AN, Platt ML (2005) Risk-sensitive neurons in macaque posterior cingulate cortex. Nat Neurosci 8:1220-1227.

McDonald AJ (1998) Cortical pathways to the mammalian amygdala. Prog Neurobiol 55:257-332.

Mobini S, Body S, Ho MY, Bradshaw CM, Szabadi E, Deakin JF, Anderson IM (2002) Effects of lesions of the orbital prefrontal cortex on sensitivity to delayed and probabilistic reinforcement. Psychopharmacology 160:290298.

Mohr PN, Biele G, Heekeren HR (2010) Neural processing of risk. J Neurosci 30:6613-6619.

Naqvi NH, Rudrauf D, Damasio H, Bechara A (2007) Damage to the insula disrupts addiction to cigarette smoking. Science 315:531-534.

O'Doherty J, Kringelbach ML, Rolls ET, Hornak J, Andrews C (2001) Abstract reward and punishment representations in the human orbitofrontal cortex. Nat Neurosci 4:95-102.

O'Neill M, Schultz W (2010) Coding of reward risk by orbitofrontal neurons is mostly distinct from coding of reward value. Neuron 68:789-800.

Pais-Vieira M, Lima D, Galhardo V (2007) Orbitofrontal cortex lesions disrupt risk assessment in a novel serial decision-making task for rats. Neuroscience 145:225-231.

Paulus MP, Rogalsky C, Simmons A, Feinstein JS, Stein MB (2003) Increased activation in the right insula during risk-taking decision making is related to harm avoidance and neuroticism. Neuroimage 19:1439-1448.

Paxinos G, Watson C (2007) The rat brain in stereotaxic coordinates, sixth edition. London: Academic.

Preuschoff K, Quartz SR, Bossaerts P (2008) Human insula activation reflects risk prediction errors as well as risk. J Neurosci 28:2745-2752.

Reynolds SM, Zahm DS (2005) Specificity in the projections of prefrontal and insular cortex to ventral striatopallidum and the extended amygdale. J Neurosci 25:11757-11767.

Rogers RD, Everitt BJ, Baldacchino A, Blackshaw AJ, Swainson R, Wynne K, Baker NB, Hunter J, Carthy T, Booker E, London M, Deakin JF, Sahakian BJ, Robbins TW (1999a) Dissociating deficits in the decision-making cognition of chronic amphetamine abusers, opiate abusers, patients with focal damage to prefrontal cortex, and typtophan-depleted normal volunteers: evidence for monoaminergic mechanisms. Neuropsychopharmacology 20:322-339.

Rogers RD, Owen AM, Middleton HC, Williams EJ, Pickard JD, Sahakian BJ, Robbins TW (1999b) Choosing between small, likely rewards and large, 
unlikely rewards activates inferior and orbital prefrontal cortex. J Neurosci 19:9029-9038.

Roitman JD, Roitman MF (2010) Risk-preference differentiates orbitofrontal cortex responses to freely chosen reward outcomes. Eur J Neurosci 31:1492-1500.

Rushworth MF, Noonan MP, Boorman ED, Walton ME, Behrens TE (2011) Frontal cortex and reward-guided learning and decision-making. Neuron 70:1054-1069.

Schilman EA, Uylings HB, Galis-de Graaf Y, Joel D, Groenewegen HJ (2008) The orbital cortex in rats topographically projects to central parts of the caudate-putamen complex. Neurosci lett 432:40-45.

Schultz W (2011) Potential vulnerabilities of neuronal reward, risk and decision mechanisms to addictive drugs. Neuron 69:603-617.

Shi CJ, Cassell MD (1998) Cortical, thalamic, and amygdaloid connections of the anterior and posterior insular cortices. J Comp Neurol 399:440-468.

Takahashi YK, Roesch MR, Stalnaker TA, Haney RZ, Calu DJ, Taylor AR, Burke KA, Schoenbaum G (2009) The orbitofrontal cortex and ventral tegmental area are necessary for learning from unexpected outcomes. Neuron 62:269-280.

Tobler PN, O'Doherty JP, Dolan RJ, Schultz W (2007) Reward value coding distinct from risk attitude-related uncertainty cording in human reward systems. J Neurophysiol 97:1621-1632.

Tom SM, Fox CR, Trepel C, Poldrack RA (2007) The neural basis of loss aversion in decision-making under risk. Science 315:515-518.

Tversky A, Kahneman D (1981) The framing of decisions and the psychology of choice. Science 211:453-458.

Van De Werd HJ, Uylings HB (2008) The rat orbital and agranular insular prefrontal cortical areas: a cytoarchitectonic and chemoarchitectonic study. Brain Struct Funct 212:387-401.

Venkatraman V, Payne JW, Bettman JR, Luce MF, Huettel SA (2009) Separate neural mechanisms underlie choices and strategic preferences in risky decision making. Neuron 62:593-602.

Weber EU, Shafir S, Blais AR (2004) Predicting risk sensitivity in humans and lower animals: risk as variance or coefficient of variation. Psychol Rev 111:430-445

Winstanley CA, Theobald DE, Cardinal RN, Robbins TW (2004) Contrasting roles of basolateral amygdala and orbitofrontal cortex in impulsive choice. J Neurosci 24:4718-4722.

Xue G, Lu Z, Levin IP, Bechara A (2010) The impact of prior risk experiences on subsequent risky decision-making: the role of the insula. Neuroimage 50:709-716. 